\title{
Revised exon-intron structure of human JAK3 locus
}

\author{
Rik A Brooimans, Annemarie J van der Slot, Adriënne JAM van den Berg and \\ Ben JM Zegers
}

Department of Pediatric Immunology, Wilhelmina Children's Hospital, University Medical Center Utrecht, The Netherlands

\begin{abstract}
Jak3, a member of the Janus tyrosine kinase family is an intracellular kinase functionally coupled to cytokine receptors that share a common $\gamma$ chain $\left(\gamma_{c}\right)$. Defects in the $\gamma_{c}$ or Jak3 result in $\mathrm{T}^{-} \mathrm{B}^{+}$severe combined immunodeficiency (SCID). In order to clarify discrepancies between earlier reported genomic organisations of human $J A K 3$, the present study was undertaken to redefine its whole exon-intron structure. The genomic structure of human $J A K 3$ consists of 23 exons and 22 introns, and shows strong homology with the organisation of the murine JAK3 locus. The exon-intron sequences provided in this report can be used to facilitate the identification of new Jak3-deficient SCID patients, including prenatal diagnosis.
\end{abstract}

Keywords: JAK3; gene structure; gene organisation; SCID; immunodeficiency

\section{Introduction}

The Janus protein tyrosine kinase (Jak) family plays a fundamental role in cytokine receptor signal transduction pathways of several haematopoietic cytokines. ${ }^{1,2}$ To date, this family consists of four members, Jak1, Jak2, Jak3 and Tyk2. In contrast to the other members, which have ubiquitous tissue expression, Jak 3 is preferentially expressed in lymphoid and myeloid cells. ${ }^{3}$ Jak3 is physically associated with the cytokine receptor common $\gamma$ chain $\left(\gamma_{c}\right)$, a receptor subunit shared between the receptors for IL-2, IL-4, IL-7, IL-9, and IL-15. ${ }^{4,5}$ Recently, mutations affecting the expression of

Correspondence: Dr RA Brooimans, Department of Pediatric Immunology, Wilhelmina Children's Hospital, University Medical Center Utrecht, PO Box 85090, 3508 AB Utrecht, The Netherlands. Tel: + 3130 2504354; Fax: + 3130 2505311; E-mail: R.Brooimans@wkz.azu.nl

Received 15 February 1999; revised 12 May 1999; accepted 8 June 1999
Jak3 were found to be responsible for autosomal recessive severe combined immunodeficiency (SCID) in humans. ${ }^{6,7}$ Patients with SCID due to Jak3 deficiency (Jak3-SCID) have a clinical phenotype that is similar to the X-linked variant of SCID that is caused by mutations of $\gamma_{c}$. Both forms of SCID patients are characterised by persistent severe infections in early life, lack of T cells and NK cells and normal to elevated numbers of nonfunctional B cells. The JAK3 gene has been localised to human chromosome 19p13.1 and its genomic organisation was characterised by two research groups. ${ }^{8,9}$ Comparison of both reports revealed several discrepancies between the two published structures, including differences in the number of exons that encodes $J A K 3$. In this paper, we report on a revised genomic structure of the human $J A K 3$ gene, including detailed information of the exon-intron boundary sequences that allows development of PCRbased mutation analysis for identification of new patients. 


\section{Materials and Methods}

\section{Amplification and Nucleotide Sequence \\ Determination}

DNA fragments up to $6.0 \mathrm{~kb}$ were amplified from human high molecular weight DNA, using the long and accurate PCR technology (Takara Biomedicals, Gennevilliers, France) according to the manufacturer's instructions with primers based on either the coding region of $J A K 3^{3}$ or identified intron sequences. The PCR products were sequenced using the AmplitaqCycle Sequencing kit (Perkin Elmer Applied Biosystems, Langen, Germany) following manufacturer's instructions. The flanking regions of the exons found were used to develop primers in order to amplify the exons separately.

\section{Results}

To define the whole exon-intron structure we amplified the entire JAK3 gene using a long template PCR method. Several pairs of overlapping primers were designed in the coding sequence that encompasses the whole human $J A K 3$ gene. The PCR fragments obtained were compared in length with the published $J A K 3$ sequences. The most striking difference observed concerned the fragment length of the amplified $\mathrm{C}$ terminal end of $J A K 3$ between exon 18 and the 3 ' untranslated region. According to the sequence by Riedy ${ }^{8}$ a fragment of $1024 \mathrm{bp}$ was estimated, but a specific fragment of around $5000 \mathrm{bp}$ was amplified. Sequence analysis of this fragment revealed the presence of three additional exon and intron structures. Another difference in exon organisation was found at exon 7. An additional exon was identified, already described by Villa et al. ${ }^{9}$ A schematic representation of the $J A K 3$ gene in relation to the encoded Janus homology $(\mathrm{JH})$ domains of the protein has been outlined in Figure 1. JH1, the catalytic domain of $J A K 3$ is encoded by exons $17-23$. To allow the amplification of single exons with intronic primers for future mutation analysis, we sequenced 40-50 bp of intronic sequences flanking the exon-intron boundaries, as shown in Table 1.

\section{Discussion}

The genomic structure of human $J A K 3$ we identified consists of 23 exons and 22 introns (Figure 1), and shows strong homology with the organisation of the murine Jak3 locus, ${ }^{10}$ suggesting a conserved maintenance through evolution. All the splice junctions show the invariant GT/AG dinucleotides (Table 1). It has been suggested that the pseudokinase (JH2) and kinase (JH1) domains of JAK3 might have evolved from a common ancestor. Comparison of the genomic structure of the two domains demonstrates that each has a distinct organisation (Figure 1). This lack of similarity suggests that the $\mathrm{JH} 1$ and $\mathrm{JH} 2$ domains arose from different ancestral genes. In a previous study, three different splice variants ( $\mathrm{S}, \mathrm{B}$, and $\mathrm{M}$ forms) of human $J A K 3$ were found to be expressed in cells of haematopoietic and epithelial origins. ${ }^{11}$ These isoforms contain identical 5 ' sequence but diverge at the $3^{\prime}$ end. The $\mathrm{S}$ form corresponds to the published $J A K 3 \mathrm{cDNA}$ sequence, ${ }^{3}$ whereas the $\mathrm{B}$ form lacks the kinase domain. Alignment of the B form sequence with the intron sequences we identified, revealed homology between the 5' site of intron 22 and the 3 ' end of the B isoform. Thus, the B isoform does not use the splice donor site of intron 22 and generates a read-through transcript. The

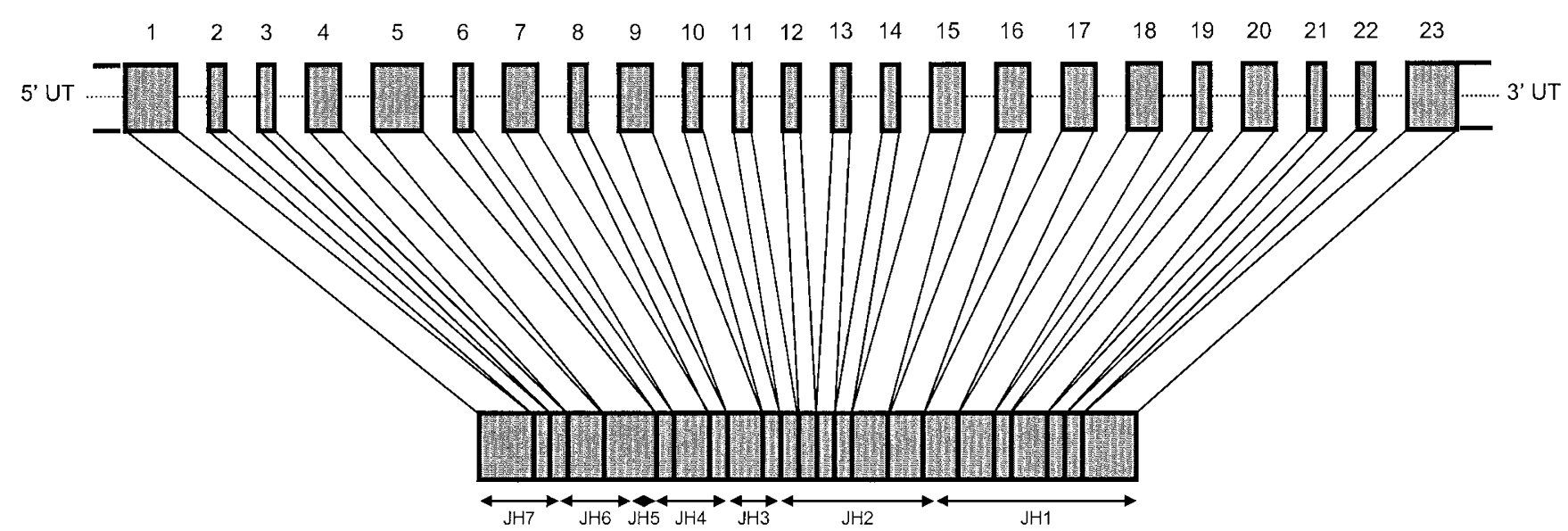

Figure 1 Exon-intron organisation of human JAK3 as related to the functional domains of the protein. The boxes represent the exons, whilst the dashed line represents introns (not in scale). The positions of the JAK homology domains JH1-JH7 are indicated at the bottom 


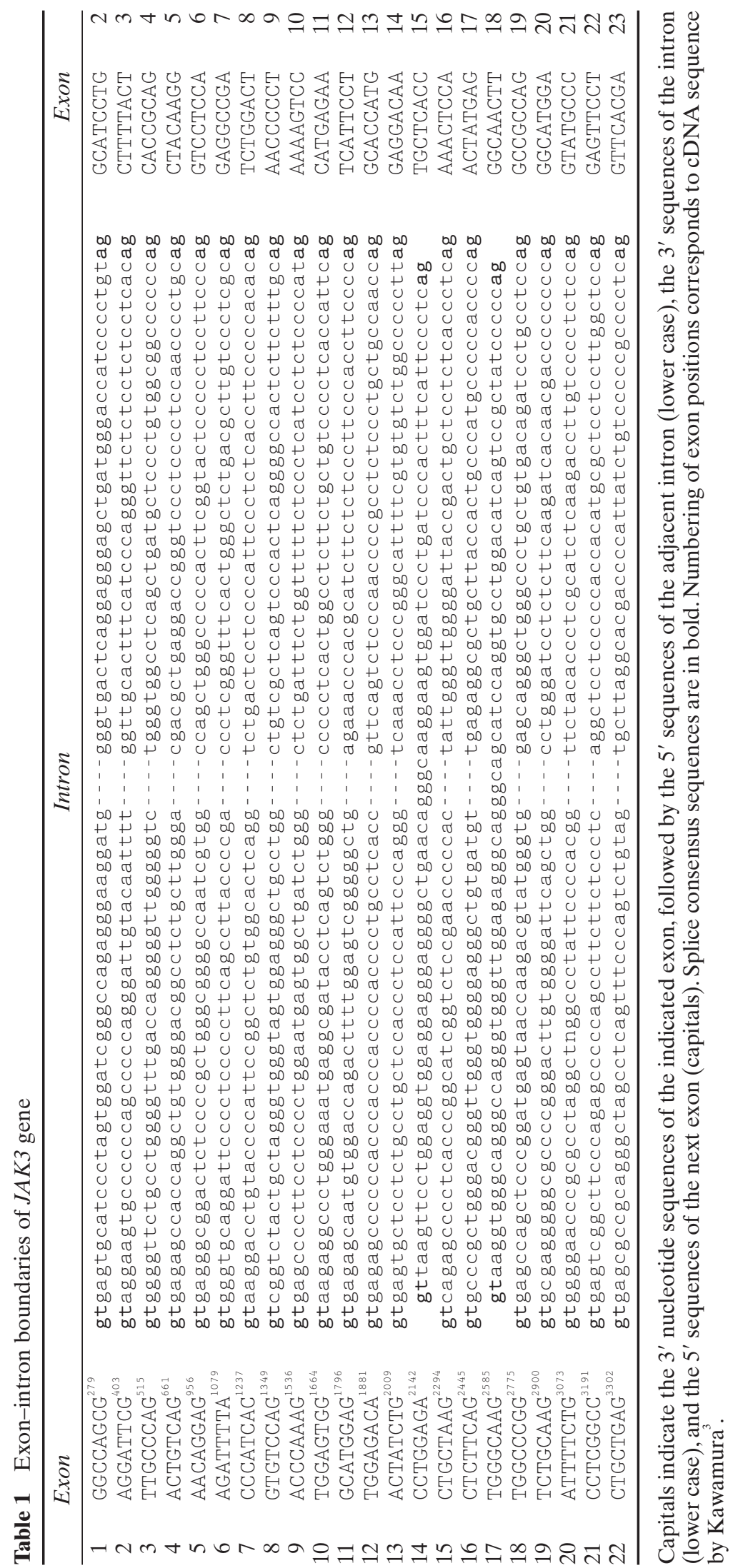


$3^{\prime}$ region of the $\mathrm{M}$ form shows no significant homology with the genomic human sequence.

The reported findings of various mutations in the $J A K 3$ gene resulting in a SCID phenotype in humans demonstrates the importance of Jak3 in cytokine signal transduction and lymphoid cell development. ${ }^{6,7,12}$ Jak3 expression is restricted to haematopoietic cell lineage; therefore it is necessary to perform prenatal diagnosis at the genomic DNA level, including carrier testing in the families concerned. The results reported here will facilitate identification of mutations in future Jak3 deficient SCID patients and may be useful to gain more insight into the molecular basis of the defect.

Reported sequences have been deposited in the EMBL database (accession nos AJ224652, AJ224653, AJ224654, AJ224655, AJ224656, AJ224657).

\section{References}

1 Ihle JN: The Janus protein tyrosine kinase family and its role in cytokine signaling. Adv Immunol 1995; 60: 1-35.

2 Taniguchi T: Cytokine signaling through nonreceptor protein tyrosine kinases. Science 1995; 268: 251-255.

3 Kawamura M, McVicar DW, Johnston JA et al: Molecular cloning of L-JAK, a Janus family protein-tyrosine kinase expressed in natural killer cells and activated leukocytes. Proc Natl Acad Sci USA 1994; 91: 6374-6378.
4 Johnston JA, Kawamura M, Kirken RA et al: Phosphorylation and activation of the Jak-3 Janus kinase in response to interleukin-2. Nature 1994; 370: 151-153.

5 Witthuhn BA, Silvennoinen O, Miura O et al: Involvement of the Jak-3 Janus kinase in signalling by interleukins 2 and 4 in lymphoid and myeloid cells. Nature 1994; 370 153-157.

6 Macchi P, Villa A, Giliani S et al: Mutations of Jak-3 gene in patients with autosomal severe combined immune deficiency (SCID). Nature 1995; 377: 65-68.

7 Russell SM, Tayebi N, Nakajima H et al: Mutation of Jak3 in a patient with SCID: essential role of Jak3 in lymphoid development. Science 1995; 270: 797-800.

8 Riedy MC, Dutra AS, Blake TB et al: Genomic sequence, organization, and chromosomal localization of human JAK3. Genomics 1996; 37: 57-61.

9 Villa A, Sironi M, Macchi P et al: Monocyte function in a severe combined immunodeficient patient with a donor splice site mutation in the Jak3 gene. Blood 1996; 88 817-823.

10 Kumar A, Toscani A, Rane S, Reddy EP: Structural organization and chromosomal mapping of JAK3 locus. Oncogene 1996; 13: 2009-2014.

11 Lai KS, Jin Y, Graham DK, Witthuhn BA, Ihle JN, Liu ET: A kinase-deficient splice variant of the human JAK3 is expressed in hematopoietic and epithelial cancer cells. $J$ Biol Chem 1995; 270: 25028-25036.

12 Candotti F, Oakes SA, Johnston JA et al: Structural and functional basis for JAK3-deficient severe combined immunodeficiency. Blood 1997; 90: 3996-4003. 